International Journal of Clinical Therapeutics and Diagnosis (IJCTD)

ISSN 2332-2926

\title{
A Study on Parental Attitude and Needs of the Parents Having Children with Intellectual Disability
}

Subhash D Wagh ${ }^{1 *}$, Showkat A Ganaie ${ }^{2}$

Research Article

${ }^{1}$ Research Scholar: Department of Rehabilitation Psychology, National Institute for the Mentally Handicapped, Manovikas Nagar, Secunderabad, An dra Pradesh-500009,India.

${ }^{2}$ Contractual Lecturer: Post Graduate Department of Psychology, University of Kashmir, Hazratbal J\&K India - 190006

\begin{abstract}
The present study aimed to assess and understand the attitude and needs of the parents having children with intellectual disability. Thirty parents (both father \& mother) were including in the sample from general services of National Institute for the mentally handicapped, Secunderabad. The assessment was done by using "A Scale to measure, parental attitude towards mental retardation (Rangswami, 1986) and NIMH-FAMNS (Parents) to measure needs of the parents". In the present study Data was analyzed by using statistical package for social sciences (SPSS 17.0). Mean, S.D, independent " $t$ " test, ANOVA, and Pearson correlation was calculated. The results of study indicated that Disability in children gives rise to various needs in parents, which may vary according to the nature of disability and parental characteristic one among these characteristics is attitude. Attitude and needs are interrelated; when parental attitude is positive towards their children they express more concern and needs. In present study, parents of children with moderate mental retardation showed high positive attitude than mild and severe. The majority of parents showed medium positive attitudes toward their children with intellectual disability. Further researches are needed to conduct on large sample which will help towards better generalization.
\end{abstract}

Keywords: Attitude, Needs of parents, Intellectual disability

\section{*Corresponding Author:}

Subhash D Wagh,

Department of Rehabilitation Psychology, National Institute for the Mentally Handicapped, Manovikas Nagar, Secunderabad, Andhra

Pradesh - 500009, India.

E-mail: deealiwaga011@gmail.com

Received: August 14, 2014

Accepted: August 26, 2014

Published: August 27, 2014

Citation: Subhash D Wagh, Showkat A Ganaie (2014) A Study on Parental Attitude and Needs of the Parents Having Children with Intellectual Disability. Int J Clin Ther Diagn. 2(4), 56-58. doi: http:/ / dx.doi. org/10.19070/2332-2926-1400011

Copyright: Subhash D Wagh ${ }^{\odot} 2014$. This is an open-access article distributed under the terms of the Creative Commons Attribution License, which permits unrestricted use, distribution and reproduction in any medium, provided the original author and source are credited.

\section{Introduction}

Intellectual Disability is perceived differently by different people ranging from burdens to the family to productive members of the society. Though Intellectual Disability is a condition such as visual, hearing, or orthopedic disabilities it is less understood or misunderstood because of its inconspicuous nature, while the other disabilities are obvious when we look at the affected person. A mentally retarded person most often looks normal without physical deformities and therefore people have difficulty in understanding while he acts differently from others.According to American Association on Intellectual and Developmental Disabilities (AAIDD-2010), "Intellectual Disability is a disability characterized by significant limitations both in intellectual functioning and in adaptive behavior, which covers many everyday social and practical skills. This disability originates before the age of 18."

\section{Concept and Definition of Attitude}

The term 'attitude' is used loosely in the literature to cover parent behavior, perceptions, reactions, values, feelings etc. Attitude can be considered a moderately intense emotion that prepares or pre- disposes an individual to respond consistently in a favorable or unfavorable manner when confronted with a particular object. Attitude is defined as' a mental and neural state of readiness, organized through experiences exerting directive or dynamic influence upon the individual's response to all objects and situations with which it is related (Allport, 1954)

All parents show one of three basic responses towards their retarded child. First, the child is accepted for what he is as he is, and the parents recognizing the child limitations try to the best of their abilities to provide the most wholesome environment possible. Second, the parents either accept or reject the child, with possible shading of acceptance and rejection reaction, but decided to institutionalize him, Third, they reject the child either part or in whole (Ehlers, Krishef, \& Prothern1977).

\section{Needs of the parents}

The presence of a child with Intellectual Disability in the family calls for a lot of adjustment on the part of the parents and the family members (Peshawaria \& Menon, 1991). Some families cope better with the situation than others. Research has indicated that approaches that focus on meeting needs of all members of the family are more effective in helping the family to cope with the situation than approaches that focus only on the child with Intellectual Disability. Identifying and supporting the parents in their efforts to meet the needs of all the family members (including themselves) is one of the most efficient ways of developing 
parental skills which can enhance the development of all family members. Research has also indicated that greater the number of unmet needs, greater is the number of emotional and physical problems reported by the parents (Dunset \& Leet, 1987).

Assessment of family needs has become imperative with the universal adoption of the international classification of functioning and disability and health (WHO 2001), as it sees a person's functioning and disability as a dynamic interaction between health condition and contextual factors. Contextual factors include personal and environmental factors such as sex, age, health conditions, upbringing, coping styles, social background and formal and informal social structures. Understanding how the nature of needs of parents having children with Intellectual Disability changes over time would enable service providers to design appropriate support services.

In the present study the term needs of the parents refers to the needs related to the condition of the child, needs related to the management of the children, needs related to the facilitation of interaction, services, emotional and social needs, physical supports, financial support, family relationship, future planning and needs related to government benefits and legislation of the parents having children with Intellectual Disability.

Disability in children gives rise to various needs in parents, which may vary according to the nature of disability and parental characteristics one among these characteristics is attitude. Attitude and needs are inter dependant, fulfilment of needs can determine attitude of a person.

Aim of the study: The aim of the study is to analyse the relationship between parental attitude and needs of the parents having children with Intellectual Disability.

Hypotheses: There will be no significant relationship between parental attitude and needs of the parents having children with Intellectual Disability

\section{Methodology}

The present study is an exploratory research with correlational design.Sample - The sample consists of 30 parents having children with Intellectual Disability. Age range - Age range of parents 25 to 50. Sampling technique- Random sampling technique was use. Sample collection - Sample was collected from the general services National Institute for the Mentally Handicapped, Secunderabad.

\section{Tool used}

1. Parental attitude scale towards persons with Intellectual Disability developed by K.Rangaswami at Indian institute of Mental Health, Madras (1986) was used to collect data on parental attitude. The scale consists of 45 items which covers 9 areas namely. These nine areas are; Over protection, acceptance, permissiveness, rejection, guilt and shame, self restriction, optimism, aggression and hostility, education. The items on the attitude scale were presented in the form of rating scale having three points. 'yes', 'cannot say', 'no'. For positive items the weights given were 0-1-2 in order and for negative items the weights given were 2-1-0 in order. The attitudes scores of the respondent were obtained by summing up the weights for the individuals items on the scale. The maximum possible score is 90 . More the scores, greater the negative attitude. To avoid response set the items are worded in both positive and negative directions. Subscale scores can be obtained by adding the scores of sub scale items.

2. NIMH Family Needs Schedule (NIMH - FAMNS) for parents was used to assess the needs of the parents having children with Intellectual Disability. This tool was developed by Peshawaria, Menon, Ganguly, Roy, Pillay and Gupta (1995).NIMHFAMNS, is a semi structured interview schedule is used for the following purposes.

1. To identify needs of the Indian families having individuals with Intellectual Disability.

2. To priorities the needs for family intervention.

3. To objectively evaluate family intervention programme.

The NIMH Family needs schedule comprises of 15 areas. Among the 15 areas the investigator selected only 11 areas such as information condition, child management, personal emotional, personal social, facilitating interaction, services, physical support, financial support, family relationship, future planning and government benefits and legislation. All the remaining 4 areas looked for the adult with Intellectual Disability. The area information condition consists of 6 items, child management area consists of 8 items, facilitating interaction consists of 2 items, services consists of 5 items, personal emotional consists of 4 items, personal social consists of 2 items, physical support consists of 3 items, financial support consists of 3 items, family relationship area consists of 2 items, future planning area include 2 items and last government benefits and legislation consists of 2 items.

\section{Procedure of Data Collection}

Written consent was taken from the department and institute for data collection. Rapport was established with participants and each of them was provided with both the scales. A brief demographic detail was also obtained from them. Both the tools were self administered tools. Both tools were administered on parents having children with intellectual disability at a time. The instructions were given to the participants "please carefully go through the instruction before proceeding with the scales, and there is no right or wrong response for the items. Your responses would be kept confidential". If they had any problems to understand the clarifications were given.

\section{Data Analysis}

The data analysis was done using Statistical Package for Social Sciences 20 version for windows (SPSS 17).Computation of mean, standard deviation and percentage use for assessed the variables (child gender, age, level of retardation, etc Correlation was used to find the relationship between parental attitude and needs of the parents having children with intellectual-disability.

\section{Result and Discussion}

Table-1 shows the demographic details of children with Intellectual Disability. Male are $20(66.7 \%)$ and female are $10(33.3 \%)$. It is observed that with age range of 6 to 18 years is $18(60 \%)$, children with age range of 19 to 25 years are 12 (40\%). Children are categorized under three levels of Intellectual Disability. Children having mild Intellectual Disability are 11(36.7\%), moderate Intellectual Disability is $10(33.3 \%)$ and severe Intellectual Disability is $9(30 \%)$. 
Table- 2 shows the demographic details of parents having children with Intellectual Disability. Urban are $18(60 \%)$ and rural are 12 (40\%). Parents having nuclear family are $21(70 \%)$ and parents having joint family are $9(30 \%)$.

Table- 3 shows that the inter correlation between family needs and parental attitude is high. There is a significant positive relationship between parental attitude and needs of the parents having children with Intellectual Disability, thus the null hypothesis, there will be no significant relationship between parental attitude and needs of the parents having children with Intellectual Disability is rejected at level $(p<0.05)$. The present study found that, the high positive attitude and needs of parents having children with Intel- lectual Disability. Present study also found that parents having medium positive attitude towards their children with mild and severe Intellectual Disability. Parental needs like; knowing about the child, getting information on dealing with the emotions of the child, training for the discipline which is acceptable by the society, services, and government benefits. The findings further revealed that parents having high positive attitude towards the children with moderate Intellectual Disability expressed needs like; training the children in activities of daily living, education, planning their child's future and understanding their child's legal rights. The main finding of the study showed that there is a significant relationship between parental attitude and needs of the parents. Parents having children with Intellectual Disability also having high positive attitude then their needs will be more. There are

Table 1. Demographic details of the children with Intellectual Disability $(\mathbf{N}=30)$

\begin{tabular}{|c|c|c|c|c|}
\hline$[1]$ & Variable & Category & 'n' & percent \\
\hline \multirow[t]{2}{*}[2]{} & \multirow[t]{2}{*}{ Gender } & Male & 20 & 66.7 \\
\hline & & Female & 10 & 33.3 \\
\hline \multirow[t]{2}{*}[3]{} & \multirow[t]{2}{*}{ Age } & (6-18)yrs & 18 & 60.0 \\
\hline & & $(19-25)$ yrs & 12 & 40.0 \\
\hline \multirow[t]{3}{*}[4]{} & \multirow[t]{3}{*}{ Level of Intellectual Disability } & Mild & 11 & 36.7 \\
\hline & & Moderate & 10 & 33.3 \\
\hline & & Severe & 9 & 30.0 \\
\hline
\end{tabular}

Table 2. Demographic details of the parents having children with Intellectual Disability

\begin{tabular}{|l|l|l|l|}
\hline Variable & Category & 'n' & percentage \\
\hline \multirow{3}{*}{ Locality } & Urban & 18 & 60.0 \\
\cline { 2 - 4 } & Rural & 12 & 40.0 \\
\hline \multirow{2}{*}{$\begin{array}{l}\text { Type of fam- } \\
\text { ily }\end{array}$} & Nuclear & 21 & 70.0 \\
\cline { 2 - 4 } & Joint & 9 & 30.0 \\
\hline
\end{tabular}

Table no 3: Pearson correlation between parental attitude and needs of the parents Pearson correlation

\begin{tabular}{|l|l|l|}
\hline Variables & N & Pearson 'r' \\
\hline Parental attitude Vs Family needs & 30 & $\begin{array}{l}\text { 'r' }=0.439 * \\
\mathrm{p}<0.05, \\
\text { Significant }\end{array}$ \\
\hline
\end{tabular}

few studies which are supporting and/or contradictory to present findings. The parents are the first and most important teachers for children, as they play the role of teaching during interaction with children (Lin, 1996)

\section{Conclusion}

The present research study concluded that the higher the parental needs of parents, the higher the parental attitude of parents having children with Intellectual Disability and most of them are having medium positive attitude towards their children with mild and severe Intellectual Disability. The study revealed that there is a significant relationship between parental attitude and needs of the parents.

\section{References}

[1]. Akhtar S, Verma V.K. (1972). Guidelines for parents of the mentally retarded children. Indian journal of Intellectual Disability 5(2):75.

[2]. Allred K.W, Cooper C.S. (1991) A Comparison of mothers versus fathers needs for support in caring for a young child with special needs InfantToddler Intervention 2:205-221.

[3]. Altman B (1981). Studies of attitudes towards the handicapped: The need for a new direction. Social problems, 28: 321-337.

[4]. Shogren KA, Turnbull HR (2010) Public policy and outcomes for persons with intellectual disability: extending and expanding the public policy framework of AAIDD's 11th Edition of Intellectual Disability: Definition, Classification, and Systems of Support. Intellect Dev Disabil 48(5):375-86

[5]. Bailey D.B, Blasco P.M, Simeonsson R.J (1992) Needs expressed by mother and fathers of young children with disabilities. American Journal of Intellectual Disability, 97:1-10.

[6]. Ramgopal C.N (1988) A study on behviour disorders in moderate mental retarded children and its relation to parental attitude. M.Phil Dissertation submitted to MIMHANS, Bang Lore University.

[7]. Paswan A.D (2007) parental attitude towards their children with Intellectual Disability in Srinagar M.Phil Dissertation submitted to NIMH, Secunderabad.

[8]. Peshawaria R, Menon D.K (1991) theory and practice - working with the families of children with mental handicap in India. Secunderabad: NIMH.

[9]. Ramgopal C.N (1988) A study on behviour disorders in moderate mental retarded children and its relation to parental attitude. M.Phil Dissertation submitted to MIMHANS, Bang Lore University.

[10]. Trevino F (1970) Sibling of handicapped children: identifying those at risk. Social case work pp.60.

[11]. Varma K, Kishore M.T (2009) Needs of Indian Parents having children with intellectual disability. International Journal of Rehabilitation research 32:71-76

[12]. World Health Organization (2001). International classification of functioning disability and Health: ICF.Geneva: WHO 